\title{
Medición de niveles de colinesterasas eritrocitarias en agricultores usuarios de plaguicidas y en practicantes de agroecología, San Cristóbal, Medellín, Colombia
}

\author{
Measurement of erythrocyte cholinesterase levels in farmers who use pesticides \\ and agroecology practitioners from San Cristóbal, Medellín, Colombia
}

\section{Medição dos níveis de colinesterases eritrocitárias em cultivadores usuários de pesticidas e profissionais de agroecologia, San Cristóbal, Medellín, Colômbia}

Ysabel Polanco-López-de-Mesa¹, David Hernández-Carmona², Martha Lucía Escobar-Pérez, Daniel Camilo AguirreAcevedo ${ }^{4}$; Ánderson Parra-Hernández 5 .

1 Doctora en Salud Pública, médica, Departamento de Medicina Preventiva y Salud Pública, Facultad de Medicina, Universidad de Antioquia. Colombia. ysabel.polanco@udea.edu.co. oRcID: orcid.org/0000-0003-2616-0160.

2 Maestría en Salud Colectiva, historiador, investigador Facultad Nacional de Salud Pública, Universidad de Antioquia, Colombia. hernandez.davidc@gmail.com. ORCID: orcid.org/0000-0003-4513-6406.

3 Maestría en Salud Colectiva, médica, Departamento de Medicina Preventiva y Salud Pública, Facultad de Medicina, Universidad de Antioquia, Colombia. martha.escobar@udea.edu.co. oRcID: orcid.org/0000-0001-7407-9653.

4 Doctor en Epidemiología, estadístico, Departamento de Educación Médica, Facultad de Medicina, Universidad de Antioquia, Colombia. daniel.aguirre@udea.edu.co. oRCID: orcid.org/0000-0002-8195-8821.

5 Estudiante de Medicina, Universidad de Antioquia, Colombia. anderson.parrah@udea.edu.co. orcID: orcid.org/0000-0001-6380-5007.

Recibido: 16/07/2018. Aprobado: 13/06/2019. Publicado: 01/10/2019

Polanco-López-de-Mesa Y, Hernández-Carmona D, Escobar-Pérez ML, Aguirre-Acevedo DC, Parra-Hernández A. Medición de niveles de colinesterasas eritrocitarias en agricultores usuarios de plaguicidas y en practicantes de agroecología, San Cristóbal, Medellín, Colombia. Rev. Fac. Nac. Salud Pública. 2019;37(3):25-33. Dor:10.17533/udea.rfnsp.v37n3a04

\section{Resumen}

Objetivo: analizar el valor de las colinesterasas eritrocitarias como biomarcador de exposición a plaguicidas (organofosforados y carbamatos) y los posibles efectos en la salud de sus usuarios y de quienes practicaban la agroecología en el corregimiento de San Cristóbal, Medellín, Colombia. Metodología: Estudio de corte. A una muestra de 40 agricultores se les realizó medición de este biomarcador. Se relacionó con información demográfica, descripción de las prácticas agrícolas y examen clínico. Resultados: Según los niveles de colinesterasa eritrocitaria en individuos que usan o no plaguicidas, los resultados mostraron un valor significativo en los que no los emplean $(p=0,042)$. No se presentaron resultados significativos $(p>0,05)$ entre las mujeres que usaron plaguicidas que alteran las colinesterasas y las que no, mientras que en los hombres sí lo fueron $(p<0,032)$. Sin embargo, no hubo diferencias estadísticamente significativas 
en las colinesterasas eritrocitarias entre el grupo de los usuarios de plaguicidas y los practicantes de la agroecología. Un hallazgo importante fue el deficiente uso del equipo de protección personal y la poca supervisión técnica acerca del uso adecuado de plaguicidas. No se encontraron síntomas asociados con intoxicación por plaguicidas al momento de recolección de la información. Conclusiones: Los usuarios de plaguicidas inhibidores de las colinesterasas tuvieron niveles de colinesterasas eritrocitarias más bajos que quienes practicaban la agroecología. El uso de equipo protector fue deficiente. Por todo esto, esta población debería mejorar las medidas de protección y fomentar prácticas agrícolas más amigables con la salud y el medio ambiente, como la agroecología. --Palabras clave: plaguicidas, agricultores, colinesterasas eritrocitarias, agroecología, San Cristóbal (Medellín, Colombia).

\section{Abstract}

Objective: To analyze erythrocyte cholinesterase values as biomarkers of exposure to pesticides (organophosphates and carbamates) and their possible effects on their users and agroecology practitioners' health in the village of San Cristóbal, Medellín, Colombia. Methodology: Cross-sectional study in which a sample of 40 farmers was measured to see the presence of this biomarker. The measurement was then compared to demographic information, description of agricultural practices, and clinical examinations. Results: Depending on the erythrocyte cholinesterase levels in subjects who use or do not use pesticides, results showed a significant value in those who do not use them $(p=0.042)$. Results between women using pesticides modifying cholinesterase levels and those not using them were not statistically significant $(\mathrm{p}>0.05)$. On the contrary, results in men were significant $(p<0.032)$. However, no statistically significant differences in erythrocyte cholinesterase levels were found between the groups of pesticide users and agroecology practitioners. An important finding was a deficient use of personal protective gear and poor technical supervision of proper use of pesticides. No symptoms associated with pesticide poisoning were found at the moment of data collection. Conclusions: Pesticides users inhibiting cholinesterase presented lower cholinesterase erythrocyte levels compared to those who practiced agroecology. The use of protection gear was poor. Therefore, this population should improve protection measures and promote more health and environmentally friendly agricultural practices, such as agroecology.

--------Keywords: pesticides, farmers, erythrocyte cholinesterase, agroecology, San Cristóbal (Medellín, Colombia)

\section{Resumo}

Objetivo: Analisar o valor das colinesterases eritrocitárias como biomarcador da exposição a pesticidas (organofosfatos e carbamatos) e os possíveis efeitos na saúde de os seus usuários e estagiários do curso agroecologia no distrito de San Cristóbal, Medellín, Colômbia. Metodologia: Estudo em metodologia de corte. Uma amostra de 40 cultivadores foi medida para esse biomarcador. Relacionou-se com informação demográfica, descrição das práticas agrícolas e exame clínico. Resultados: De acordo com os níveis de colinesterase eritrocitária em indivíduos que usam ou não pesticidas, os resultados mostraram um valor significativo naqueles que não os utilizam $(p=0,042)$. Não houve resultados significativos ( $p>$ $0,05)$ entre as mulheres que usavam pesticidas que alteram a colinesterase e as que não usavam, enquanto nos homens eram $(p<0,032)$. No entanto, não houve diferenças estatisticamente significativas na colinesterase eritrocitária entre o grupo de usuários de pesticidas e estagiários da agroecologia. Uma descoberta importante foi o uso errado do equipamento de proteção individual e a falta de supervisão técnica respeito do uso adequado de pesticidas. Não foram encontrados sintomas associados ao envenenamento por pesticidas no momento em que as informações foram coletadas. Conclusões: Usuários de pesticidas inibidores das colinesterases apresentaram níveis mais baixos de colinesterase eritrocitária do que aqueles que praticavam agroecologia. $\mathrm{O}$ uso de equipamentos de proteção foi ruim. Por tudo isso, essa população deve melhorar as medidas de proteção e promover práticas agrícolas mais amigáveis à saúde e ao meio ambiente, como a agroecologia.

-------- Palavras-chave: pesticidas, agricultores, colinesterase eritrocitária, agroecologia, San Cristóbal (Medellín, Colômbia). 


\section{Introducción}

En el transcurso del último siglo, los trabajadores agrícolas han aumentado el uso de plaguicidas para optimizar sus cultivos [1,2]. Este uso y la exposición a ellos es un problema fundamental de salud pública que afecta directamente a millones de personas en las comunidades agrícolas de todo el mundo, a las comunidades de su entorno [3-5], como también a otros miembros del hogar, principalmente los niños y las mujeres embarazadas [612]. Así mismo, impacta negativamente la base de bienes y servicios ecológicos de las localidades [4,13,14]. Esta práctica ha generado un incremento de problemas de salud de orden neurológico, inmunológico, respiratorio y reproductivo para esta población [15-19].

A pesar de la falta de estudios detallados, algunos trabajos han demostrado que esta situación de igual modo está presente en Colombia, un país con extensas prácticas agrícolas [14,20-23]. En el sector agropecuario de esta región, el uso de plaguicidas es generalizado y con frecuencia indiscriminado [14,21,23]. Además, varios estudios han encontrado alteraciones en la salud de comunidades campesinas colombianas, mostrando aumentos moderados en las tasas de prevalencia del aborto, partos prematuros, mortinatos y malformaciones congénitas en los embarazos de las esposas de los trabajadores del campo y de las mujeres trabajadoras y fumigadoras [5,24,25].

El corregimiento San Cristóbal de la ciudad de Medellín tiene una extensión de $49,54 \mathrm{~km}^{2}$ y cuenta con diecinueve veredas, de acuerdo con el perfil demográfico de San Cristóbal 2016-2020 [26]. Este corregimiento tuvo en el año 2017 una población total de 93072 habitantes. A pesar de que ha tenido un proceso de urbanización rápido, su actividad económica principal es la agricultura, realizada en parcelas familiares.

Este campo es supremamente relevante para la salud pública, ya que afecta a diferentes grupos poblacionales, incluyendo niños, niñas, mujeres en edad fértil, adultos y ancianos, y al medio ambiente [5,8,9,16,27]. Debido a esto, y teniendo en cuenta estudios previos en la población agricultora colombiana [14,20-22], el grupo investigador plantea conocer el nivel de colinesterasas eritrocitarias (CE) en agricultores del corregimiento y evaluar las diferencias entre los que usan plaguicidas y los que practican la agroecología, y relacionarlos con prácticas agrícolas, uso de equipo de protección personal (EPP) y posibles efectos a la salud.

\section{Metodología}

Se realizó un estudio de corte en el mes de junio del año 2017, en una muestra por conveniencia de 40 agricultores residentes en el corregimiento de San Cristóbal (véase Figura 1), a quienes se les tomó muestra sanguínea para medición de biomarcadores por el método de Michel, que permite evaluar las exposiciones crónicas a plaguicidas inhibidores de las CE, como organofosforados y carbamatos [28]. Los participantes fueron clasificados de acuerdo con el uso de plaguicidas o la práctica de la agroecología por autorreporte.

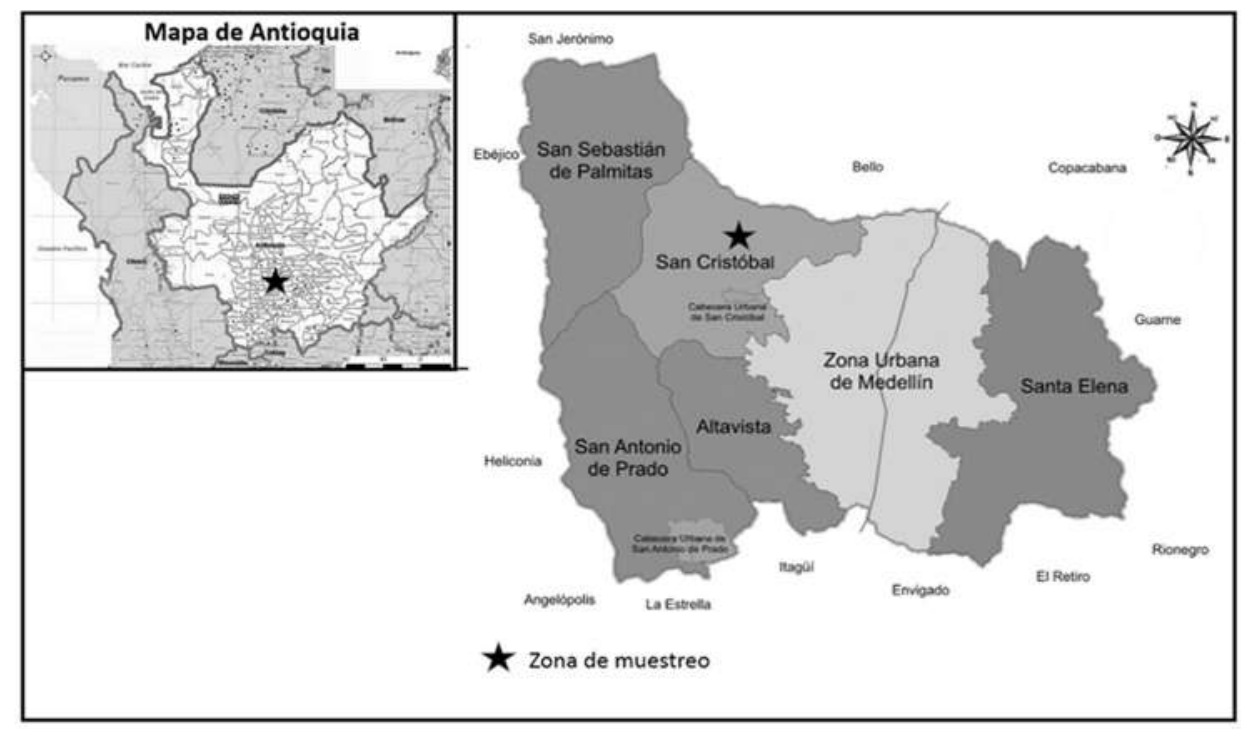

Figura 1. Zona de muestreo en el corregimiento de San Cristóbal, en el mapa de corregimientos del municipio de Medellín. Fuente: modificada por autores, de [29]. 
Mediante una encuesta estructurada, se incluyó información demográfica, se indagó por síntomas asociados a intoxicación, descripción de preparación y frecuencia de uso de las sustancias para control de plagas y el uso de EPP. Esto se realizó con el fin de comparar los resultados de la exposición.

La información se recolectó en dos sesiones, en las que los investigadores viajaron por tierra, acompañados de las auxiliares de laboratorio del Laboratorio de Toxicología de la Facultad de Medicina de la Universidad de Antioquia, quienes llevaron el material que permitía recolectar las muestras de sangre para analizar las CE.

La primera sesión fue en la vereda Travesías, y la segunda, en la vereda el Llano. Después de cada sesión, el equipo investigador se trasladó a Medellín y las auxiliares de laboratorio fueron directamente al Laboratorio de Toxicología para analizar las muestras.

Aproximadamente a las dos semanas se enviaron los resultados de laboratorio de $\mathrm{CE}$ al correo electrónico de la investigadora principal, quien posteriormente realizó entrega personalmente de estos resultados a los participantes.

Las variables cualitativas como sexo, estado civil, nivel de estudio, uso de equipos de protección y capacitación sobre protección se presentan con frecuencia absoluta y porcentaje (\%). Para las variables cuantitativas, como la edad en años y los niveles de colinesterasas $\Delta \mathrm{pH}$ por $\mathrm{mL}$ por hora $(\Delta \mathrm{pH} / \mathrm{h})$, se calcularon la media y la desviación estándar. Se valoró el supuesto de distribución normal con la prueba de Shapiro Wilk.

Se compararon los grupos según el uso o no de plaguicidas en sus características demográficas y en los niveles de CE, utilizando la prueba Chi cuadrado $\left(\chi^{2}\right)$ para las variables categóricas y la prueba $t$ de Student para muestras independientes en las variables cuantitativas.

Se realizó un análisis de sensibilidad, clasificando a los participantes dependiendo del uso de plaguicidas que alteran los niveles de colinesterasa. Mediante un modelo lineal generalizado, se tuvieron en cuenta la edad, el sexo y el uso de equipo protector como covariables, cada una evaluada de manera independiente, dadas las limitaciones con el tamaño de la muestra. Se asumió como nivel de significación un valor $p<0,05$. Los análisis fueron realizados en el IBM SPSS statistics 23,0 (IBM, versión 23, New York, Estados Unidos).

Este estudio fue aprobado por el Comité de Ética Médica de la Facultad de Medicina de la Universidad de Antioquia, en el Acta 010 del 23 de junio de 2016. Se explicó el consentimiento informado y se procedió a recolectar la información a quienes aceptaron voluntariamente su participación.

\section{Resultados}

La Tabla 1 presenta las características demográficas de los 40 agricultores estudiados, donde 21 reportaron prácticas agroecológicas y 19 usaban plaguicidas. La edad promedio fue de 53 años. La mayoría cuenta con escolaridad media. Del total de participantes, las mujeres eran el $43 \%$ de cada grupo.

En cuanto a EPP, estos eran usados por el 85,7\% de la población, y de ellos, el 45 \% recibió capacitación en la protección y el uso de plaguicidas. No se encontraron diferencias significativas en cuanto a género, edad, estado civil y nivel de estudio, aunque se presentó un mayor número de participantes con nivel secundario en el grupo de uso de plaguicidas.

Quienes practicaban agroecología utilizaron preparaciones con productos naturales (como el ajo, el ají, estiércol de vaca), productos inorgánicos (como azufre, cal, ceniza, yodo) y algunas plantas (como manzanilla, ruda, ortiga, entre otras). Por su parte, quienes usaban plaguicidas, refirieron el uso de carbamatos, organoclorados, organofosforados y piretroides.

La Tabla 2 muestra el nombre y tipo de plaguicidas inhibidores de colinesterasa (organofosforados y carbamatos) utilizados por los participantes.

El Decreto 1843 de 1991 del Invima, en su artículo 14, establece las siguientes categorías para efectos de la clasificación toxicológica de los plaguicidas en Colombia:

CATEGORIA I "Extremadamente tóxicos".

CATEGORIA II "Altamente tóxicos".

CATEGORIA III "Medianamente tóxicos".

CATEGORIA IV "Ligeramente tóxicos" [30].

Aunque la mayoría emplean plaguicidas medianamente tóxicos, preocupa la utilización de tres productos Clase I y dos Clase II, teniendo en cuenta el uso inadecuado de los EPP y la baja capacitación para el cuidado de la salud de los agricultores y sus familias. Además, algunos plaguicidas son prohibidos en Colombia. A pesar de ello, se evidenció el acceso y uso de aquellos.

Con relación al uso de EPP, se encontró una diferencia significativa a favor del grupo que practica la agroecología, pero no se hallaron diferencias con relación a capacitaciones sobre el uso adecuado de EPP.

La Figura 2 muestra los niveles de colinesterasa según uso de plaguicidas o prácticas agroecológicas, y no se encontraron diferencias significativas $(p=0,118)$. Los resultados fueron similares después de ajustar de manera independiente por edad, sexo y uso de equipo protector. Esto problematiza la situación de los agricultores, en cuanto a la cercanía que tienen las parcelas, y no hay 
Tabla 1. Características demográficas de la muestra de estudio según uso o no de plaguicidas, San Cristóbal, Medellín. Colombia.

\begin{tabular}{|c|c|c|c|c|c|c|c|}
\hline \multirow{2}{*}{$\begin{array}{l}\text { Características demográficas } \\
\text { Género masculino. } \\
\text { Frecuencia (\%) }\end{array}$} & \multicolumn{2}{|c|}{$\begin{array}{l}\text { No uso plaguicida } \\
\qquad n=21\end{array}$} & \multicolumn{2}{|c|}{$\begin{array}{l}\text { Uso plaguicida } \\
\qquad n=19\end{array}$} & \multirow{2}{*}{$\begin{array}{c}\begin{array}{c}\text { Estadístico } \\
\text { Chi cuadrado } \\
\left(\mathbf{X}^{2}\right)\end{array} \\
0,002\end{array}$} & \multirow{2}{*}{$\begin{array}{l}\text { GI } \\
1\end{array}$} & \multirow{2}{*}{$\begin{array}{r}\text { Valor } p \\
0,962\end{array}$} \\
\hline & 12 & 57,1 & 11 & 57,9 & & & \\
\hline Edad años, mediana (RI) & 53,0 & 22,5 & 53,0 & 19,0 & & & 0,872 \\
\hline Estado civil- Frecuencia (\%) & & & & & 5,6 & 4 & 0,232 \\
\hline Soltero & 5 & 25,0 & 6 & 31,6 & & & \\
\hline Casado & 13 & 65,0 & 8 & 42,1 & & & \\
\hline Viudo(a) & 0 & 0,0 & 3 & 15,8 & & & \\
\hline Convive & 0 & 0,0 & 1 & 5,3 & & & \\
\hline Separado o divorciado & 2 & 10,0 & 1 & 5,3 & & & \\
\hline Nivel de estudios, frecuencia (\%) & & & & & 5,5 & 3 & 0,1136 \\
\hline Primaria & 2 & 9,5 & 0 & 0,0 & & & \\
\hline Secundaria & 12 & 57,0 & 16 & 84,2 & & & \\
\hline Pregrado & 3 & 23,8 & 3 & 15,8 & & & \\
\hline $\begin{array}{l}\text { Uso de equipo protector. } \\
\text { Frecuencia (\%) }\end{array}$ & 18 & 85,7 & 6 & 33,3 & 11,2 & 1 & 0,001 \\
\hline $\begin{array}{l}\text { Recibió capacitación protección. } \\
\text { Frecuencia }(\%)\end{array}$ & 9 & 45,0 & 9 & 52,9 & 0,23 & 1 & 0,630 \\
\hline
\end{tabular}

Gl: grados de libertad; RI: rango intercuartil (percentil 75-percentil 25).

Tabla 2. Tipos de plaguicidas utilizados que inhiben las colinesterasas, sus características químicas y categorías toxicológicas

\begin{tabular}{|c|c|c|c|}
\hline Plaguicidas & Categoría toxicológica & Uso & $\begin{array}{c}\text { Características químicas } \\
\text { del plaguicida }\end{array}$ \\
\hline \multicolumn{4}{|l|}{ Nadir $600 \AA$} \\
\hline Paration ${ }^{\circledR}$ & Clase I & \multirow[t]{3}{*}{ Insecticidas y acaricidas } & \multirow[t]{3}{*}{ Organofosforados } \\
\hline Lorsban ${ }^{\circledR}$ & \multirow{2}{*}{ Clase II } & & \\
\hline Monitor ${ }^{\circledR}$ & & & \\
\hline \multicolumn{4}{|l|}{ Malation ${ }^{\circledR}$} \\
\hline Orthene $75 \AA$ & \multirow[t]{2}{*}{ Clase III } & \multirow[t]{2}{*}{ Insecticidas y acaricidas } & \multirow[t]{2}{*}{ Organofosforados } \\
\hline Rafaga $\circledast$ & & & \\
\hline Lannate líquida ${ }^{\circledR}$ & Clase I & & \\
\hline \multicolumn{4}{|l|}{ Aguila $®$} \\
\hline Antracol $\circledast$ & \multirow{3}{*}{ Clase III } & Fungicidas & \multirow[t]{4}{*}{ Carbamatos } \\
\hline Fitoraz ${ }^{\circledR}$ & & & \\
\hline Manzate ${ }^{\circ}$ & & & \\
\hline Dithane ${ }^{\circledR}$ & Clase IV & & \\
\hline
\end{tabular}




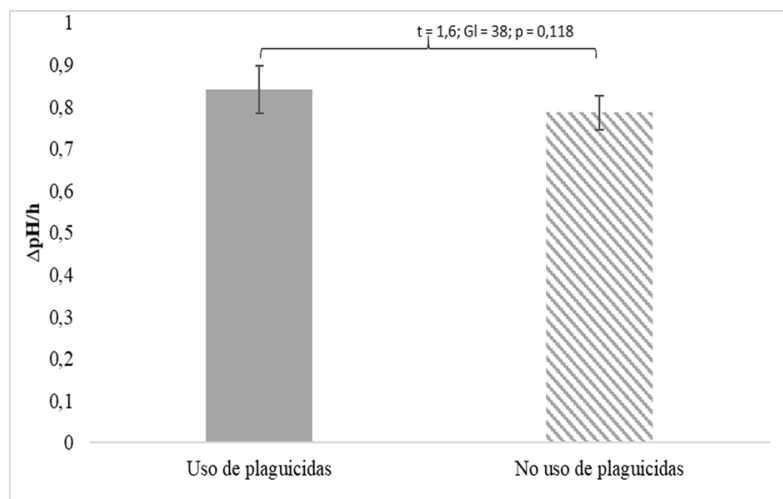

Figura 2. Niveles de colinesterasa según uso o no de plaguicidas en la población del estudio. $\Delta \mathrm{pH} /$ $\mathrm{h}=$ niveles de colinesterasas $\Delta \mathrm{pH}$ por $\mathrm{mL}$ por hora.

posibilidades de aislamiento o separación entre los que usan plaguicidas y los que practican la agroecología, principalmente por la dispersión de estos elementos en el aire y en las aguas.

Al clasificar a los individuos de acuerdo con los niveles de $\mathrm{CE}$ en dos grupos (si usan o no plaguicidas que alteran los niveles de CE), los resultados mostraron un valor significativo en el grupo que no hace uso de dichos plaguicidas $(p=0,042)$. La Tabla 3 presenta los resultados de los niveles de CE según la clasificación de uso o no de plaguicidas que las alteran, ajustados por edad, sexo y uso de equipo protector. De acuerdo con la edad, no se presentaron resultados significativos $(p=0,06)$. Según el género, en las mujeres entre las que usaron plaguicidas que alteran las CE y las que no, no hubo diferencias significativas, mientras que en los hombres, los resultados sí fueron significativos $(p<0,032)$. Los resultados en los participantes que no utilizan equipo protector fueron consistentes a favor de los que no usan plaguicidas que alteran las $\mathrm{CE}$, aunque los resultados no fueron significativos $(p=0,203)$.

\section{Discusión}

Los agricultores usuarios de plaguicidas del grupo de los organofosforados y carbamatos mostraron niveles estadísticamente significativos más bajos de CE, especialmente la población masculina, lo cual es esperable por el mecanismo de acción de estos productos y también por el hecho de que los hombres son los que más aplican los plaguicidas en estas poblaciones campesinas. Sin embargo, los agricultores que usan plaguicidas y los que hacen agroecología no tiene diferencias significativas en los valores de $\mathrm{CE}$, lo que puede deberse a múltiples causas difíciles de estandarizar.

Hay que tener en cuenta que los campesinos con prácticas agroecológicas se pueden exponer a los plaguicidas, debido a la cercanía con vecinos que sí usan plaguicidas, y en este caso, el aire puede arrastrar plaguicidas hacia ellos o el agua que consumen puede estar contaminada. Por lo tanto, es relevante insistir en las implicaciones que tienen las conductas individuales sobre la exposición, la salud de otras personas y el medio ambiente, ya que son acciones que trascienden el individuo y afectan a mayor escala.

Un estudio previo sobre prácticas agrícolas llevadas a cabo en la misma área geográfica encontró que estas implican un extenso uso de plaguicidas y una alta exposición de los campesinos agricultores [21]. Es importante investigar sobre estas prácticas agrícolas,

Tabla 3. Niveles de colinesterasa según uso o no de plaguicidas que alteran la colinesterasa ajustado por edad, sexo y uso de equipo protector.

\begin{tabular}{|c|c|c|c|c|c|}
\hline \multirow[t]{2}{*}{$\begin{array}{l}\text { Valor de colinesterasa } \\
\text { eritrocitaria* }\end{array}$} & \multicolumn{2}{|c|}{$\begin{array}{l}\text { No uso plaguicida } \\
\qquad n=21\end{array}$} & \multicolumn{2}{|c|}{$\begin{array}{l}\text { Uso plaguicida } \\
\qquad \begin{array}{c}\mathrm{n}=19 \\
\text { (Et) }\end{array}\end{array}$} & \multirow[t]{2}{*}{ Valor $p$} \\
\hline & Media & Error típico & Media & Error típico & \\
\hline $\begin{array}{l}\text { Colinesterasa, } \Delta \mathrm{pH} / \mathrm{h} \\
\text { ajustado por edad }\end{array}$ & 0,784 & 0,022 & 0,851 & 0,026 & 0,060 \\
\hline $\begin{array}{l}\text { Colinesterasa, } \Delta \mathrm{pH} / \mathrm{h} \\
\text { ajustado por sexo }\end{array}$ & 0,780 & 0,022 & 0,856 & 0,026 & 0,032 \\
\hline $\begin{array}{l}\text { Colinesterasa, } \Delta \mathrm{pH} / \mathrm{h} \\
\text { ajustado por uso de } \\
\text { equipo protector }\end{array}$ & 0,797 & 0,022 & 0,8845 & 0,027 & 0,203 \\
\hline
\end{tabular}

* El valor de referencia para colinesterasas eritrocitarias debe ser igual o mayor a: 0,874 $\Delta \mathrm{pH} / \mathrm{h}$. 
ya que los ecosistemas naturales y las comunidades campesinas agricultoras locales pueden beneficiarse significativamente del establecimiento de prácticas agrícolas no basadas en el uso de agroquímicos [2,31,32].

Es importante crear una política pública que acoja a los trabajadores del agro, ya que en Colombia las políticas se han centrado más en procesos productivos de gran escala, pero el agricultor en su minifundio ha estado más solo. A pesar de que en cada región del país se cuenta con legislación al respecto (Ley 101 de 1993 [33]), que establece la obligación de crear la Unidad Municipal de Asistencia Técnica Agropecuaria, cuya función única es la de prestar asistencia técnica agropecuaria directa y gratuita a los pequeños productores, este acompañamiento no se evidenció en la población estudiada.

Adicionalmente, la situación del EPP es compleja, ya que la mayoría de las veces el uso deficiente de estos equipos no es solo culpa de los agricultores, sino que también hay que tener en cuenta que esta indumentaria fue diseñada para ser usada solo dos horas en la mañana y dos al final de la tarde, ya que una persona no debe aplicar nunca más de 4 horas un plaguicida en el día, y esto en la práctica no siempre se cumple. Se recomienda aplicar los plaguicidas entre las 6 y las 8 a. m., y en la tarde, de 4 a 6 p. m.

Este estudio aporta al conocimiento de una realidad en una población rural cerca de una gran ciudad, y muchas veces queda oculta en los múltiples problemas de la urbe. Por esto, es importante incorporar esta población en actividades de educación rural, apoyo ocupacional e intervenciones en salud pública, que contribuyan a modificar positivamente esta realidad. Además, se requieren más investigaciones que ayuden a entender los impactos en salud asociados al uso de plaguicidas en otras regiones de Colombia, dependiendo de tipo de pesticida utilizado, el grado de exposición y la edad de las personas expuestas. Así mismo, es necesario promover la realización de estudios que aborden las implicaciones ambientales del uso de plaguicidas como elemento central de las políticas de salud pública y de la conservación, entidades estrechamente relacionadas - es decir, el vínculo entre salud humana y salud ambiental-.

En la revisión bibliográfica no se encontraron estudios comparativos entre poblaciones agricultoras que usan plaguicidas y los que practican la agroecología, y si bien este último grupo hace prácticas responsables con el ambiente, no está exento de afectarse por el uso de plaguicidas en el entorno circundante. Por esto, las personas del grupo familiar que residen en las parcelas pueden estar igualmente afectadas y se precisa investigarlas e incluirlas en estudios futuros, así como se ha hecho en otras investigaciones en otros lugares del mundo $[3,6,8,9]$.
Es importante tener muy en cuenta que las problemáticas de salud ambiental $\mathrm{y}$, en este caso, por el uso de plaguicidas en la agricultura deben trabajarse y moverse más allá del abordaje de la remediación ambiental. También hay que abordar realidades más complejas, como son las inequidades ambientales, sociales y en salud, de manera más integral y con visión a corto, mediano y largo plazo, y que sean sostenibles en el tiempo, para así propiciar cambios que puedan perdurar y realmente transformar las realidades de estas comunidades vulnerables $[1,2,34,35]$.

Así mismo, estas problemáticas de uso de plaguicidas son una muestra más de la injusticia ambiental que ocurre en estos sectores agrícolas y en estas poblaciones en Colombia, que por generaciones han estado sometidas a condiciones de vida caracterizadas por el abandono y la explotación $[1,12,36]$. Por esto, una de las opciones para disminuir la injusticia ambiental en estas poblaciones consiste en empoderar a las comunidades y construir procesos de trabajo social y ecológico que sea conjunto, participativo e interdisciplinario [12,37-41].

\section{Conclusiones}

Los usuarios de plaguicidas inhibidores de la colinesterasa tipo organofosforados y carbamatos tuvieron niveles de CE más bajos que quienes usaron otros plaguicidas, en especial los hombres de esta muestra. Adicionalmente, el uso de equipo protector fue muy deficiente. Por consiguiente, las intervenciones con la población agricultora deben ser dirigidas a mejorar las medidas de protección y a fomentar prácticas agrícolas más amigables con la salud y el medio ambiente, como la agroecología. En última instancia, se trata de entender, de manera holística, el problema del uso y la exposición a los plaguicidas en comunidades de agricultores, con el fin de mejorar a corto y largo plazo las condiciones de salud y de trabajo de las comunidades agricultoras.

Se recomienda que futuras intervenciones en salud pública y salud ambiental incorporen elementos sobre el uso apropiado de EPP, un uso apropiado de plaguicidas y ojalá una reducción paulatina de estos, con el fin de minimizar la exposición humana y la contaminación ambiental. Estos trabajos de intervención también deben incorporar participación comunitaria, con el fin de fortalecer relaciones entre los agricultores, para actuar como gremio hacia la incorporación de prácticas menos riesgosas y más justas, teniendo en cuenta que las acciones realizadas individualmente también pueden afectar a las comunidades. Quienes usan plaguicidas contaminan su parcela, así como el aire, el suelo y el agua de sus vecinos, convirtiendo esta situación en un asunto de interés y competencia tanto de la salud a nivel individual, como de la salud pública. 
Es importante fortalecer el proceso de supervisión técnica y certificación de prácticas agroecológicas, e incrementar el mercado organizado de estos productos, con el fin de que el pequeño agricultor pueda obtener mejores utilidades y satisfacer la demanda de los consumidores de productos agroecológicos. Todas estas acciones se suman al cuidado del ambiente como componente esencial de la salud y el bienestar de cada individuo y de las colectividades.

\section{Limitaciones}

Este trabajo no puede ser generalizado a todos los agricultores del corregimiento San Cristóbal de Medellín, porque la muestra fue pequeña y por conveniencia. Adicionalmente, aunque se trabajó con el grupo de agricultores agroecológicos, estos no están por completo libres de exposición a plaguicidas, ya que viven en lugares donde sus vecinos sí los usan; además, se contaminan los aires que respiran y las aguas que consumen, y algunos de ellos trabajan por jornales en parcelas de vecinos que no practican la agroecología, por lo que no se pudo obtener un ambiente totalmente libre de exposición a plaguicidas, que sería lo ideal.

La baja potencia estadística debido al tamaño de muestra limitado no permitió comparar uso o no de plaguicidas de acuerdo con el género, o por el uso o no de equipos de protección. Sin embargo, esta aproximación permite generar hipótesis sobre el riesgo al que está expuesta la población rural de Medellín.

\section{Agradecimiento}

Queremos agradecer a la organización no gubernamental Penca de Sábila y a la Asociación de Campesinos Agricultores de Boquerón por su apoyo en la coordinación de las actividades de campo y recolección de datos. También agradecemos a los campesinos de San Cristóbal, por su participación en este proyecto.

\section{Declaración de responsabilidad}

Los autores declaran que los puntos de vista expresados son responsabilidad de los autores y no de las instituciones en las que trabajan.

\section{Financiación}

La financiación la brindó el departamento de Medicina Preventiva y Salud Pública de la Facultad de Medicina de la Universidad de Antioquia.

\section{Conflicto de interés}

En este trabajo no hubo conflictos de intereses.

\section{Referencias}

1. Martinez-Alier J, Anguelovski I, Bond $\mathrm{P}$, et al. Between activism and science: Grassroots concepts for sustainability coined by Environmental Justice Organizations. J. Polit. Ecol. [internet]. 2014 [citado 2019 feb. 10]; 21(1):19. DOI: https://doi. org/10.2458/v21i1.21124. Disponible en: https:/journals.uair. arizona.edu/index.php/JPE/article/view/21124.

2. Altiery M. Agroecology the Science of Sustainable Agriculture. 2.a ed. Boca Raton: CRC Press; 2018.

3. Guillette EA, Meza MM, Aquilar MG, et al. An anthropological approach to the evaluation of preschool children exposed to pesticides in Mexico. Environ. Health Perspect. 1998;106(6):34753. DOI: https://doi.org/10.1289/ehp.98106347.

4. Quandt SA, Arcury TA, Austin CK, et al. Latino immigrants: Preventing occupational exposure to pesticides: Using participatory research with Latino farmworkers to develop an intervention. J. Immigr. Health. 2001;3(2):85-96. DOI: https:// doi.org/10.1023/A:1009513916713.

5. Nivia E. Las mujeres y los plaguicidas [internet]. Rionegro, Antioquia, Colombia; 2010 [citado 2019 mar. 8]. Disponible en: http://rapaluruguay.org/publicaciones/e_n_2010.pdf.

6. Xue J, Zartarian V, Moya J, et al. A meta-analysis of children's hand-to-mouth frequency data for estimating nondietary ingestion exposure. Risk. Anal. 2007;27(2):411-20. DOI: https://doi. $\operatorname{org} / 10.1111 / \mathrm{j} .1539-6924.2007 .00893 . x$

7. Reigart JR, Roberts JR. Pesticides in Children. Pediatr Clin. 2001;48(5):1185-98.

8. Suk WA, Ahanchian H, Asante KA, et al. Environmental pollution: An underrecognized threat to children's health, especially in low-and middle-income countries. Environ. Health Perspect. 2016;124(3):A43-5. DOI: https://doi.org/10.1289/ehp.1510517.

9. Butler-Dawson J, Galvin K, Thorne PS, et al. Organophosphorus pesticide exposure and neurobehavioral performance in Latino children living in an orchard community. NeuroToxicology [internet]. 2016 [citado 2018 oct. 3]; 53:165-72. DOI: https://doi.org/10.1016/j. neuro.2016.01.009. Disponible en: https://www.sciencedirect.com/ science/article/pii/S0161813X16300092?via\%3Dihub.

10. Bradman A, Quirós-Alcalá L, Castorina R, et al. Effect of organic diet intervention on pesticide exposures in young children living in low-income urban and agricultural communities. Environ. Health Perspect. 2015;123(10):1086-93. DOI: https://doi.org/10.1289/ ehp. 1408660.

11. Bukalasa JS, Brunekreef $\mathrm{B}$, Brouwer $\mathrm{M}$, et al. Proximity to agricultural fields as proxy for environmental exposure to pesticides among children: The piama birth cohort. Sci. Total. Environ [internet]. 2017 [citado 2018 dic. 10]; 595:515-20. Disponible en: http://dx.doi.org/10.1016/j.scitotenv.2017.03.269.

12. Schwartz NA, Von Glascoe CA, Torres V, et al. "Where they (live, work and) spray": Pesticide exposure, childhood asthma and environmental justice among Mexican-American farmworkers. Health. Place. [internet]. 2015 [citado 2018 dic. 15]; 32:83-92. Disponible en: http://dx.doi.org/10.1016/j. healthplace.2014.12.016.

13. Shiva V. Soil not Oil: Environmental Justice in a Time of Climate Crisis. Cambridge, MA: South End Press; 2009.

14. Agudelo R, Soto M, Pérez M, et al. Condiciones de vida y trabajo de familias campesinas agricultoras de Marinilla, un pueblo agrario del oriente antioqueño, Colombia, 2011. Rev. Fac. Nal. Salud Pública. [internet]. 2013 [citado 2019 feb. 12]; 31(3):31928. Disponible en: https://aprendeenlinea.udea.edu.co/revistas/ index.php/fnsp/article/view/13473/20779714. 
15. Blair A, Ritz B, Wesseling C, et al. Pesticides and human health. Occup. Env. Med. 2014;72(2):81-82. DOI: https://doi. org/10.1136/oemed-2014-102454.

16. Kim KH, Kabir E, Jahan SA. Exposure to pesticides and the associated human health effects. Sci. Total. Environ. [internet]. 2017 [citado 2018 nov. 6]; 575:525-35. Disponible en: http:// dx.doi.org/10.1016/j.scitotenv.2016.09.009.

17. Saillenfait AM, Ndiaye D, Sabaté JP. Pyrethroids: Exposure and health effects - An update. Int. J. Hyg. Environ. Health. [internet]. 2015 [citado 2018 nov. 16]; 218(3):281-92. Disponible en: http:// dx.doi.org/10.1016/j.ijheh.2015.01.002.

18. Rauh VA, Margolis AE. Research Review: Environmental exposures, neurodevelopment, and child mental health - new paradigms for the study of brain and behavioral effects. J Child. Psychol. Psychiatry. 2016;57(7):775-93. DOI: https://doi. org/10.1111/jcpp.12537.

19. Mamane A, Raherison C, Tessier J-F, et al. Environmental exposure to pesticides and respiratory health. Eur. Respir. Rev. [internet] 2015 [citado 2019 feb. 18]; 24(137):462-73. Disponible en: http:// err.ersjournals.com/lookup/doi/10.1183/16000617.00006114.

20. Sanmiguel-Valderrama $O$. The feminization and racialization labor in the Colombian fresh-cut flower industry. J. Dev. Soc. 2007;23(12):71-88. DOI: https://doi.org/10.1177/0169796X0602300205.

21. Polanco Y, Salazar J, Curbow B. A quantitative analysis of Colombian campesinos' use of pesticides: Perceived control and confidence in this use. Rev. Fac. Nac. Salud Pública. 2014;32(3):373-82.

22. Toro BM, Rojas AE, Díaz JA. Niveles de colinesterasa sérica en caficultores del departamento de Caldas, Colombia. Rev. Salud Pública [internet]. 2017 [citado 2018 feb. 3]; 19(3):318-24. DOI: https://doi.org/10.15446/rsap.v19n3.52742. Disponible en: http:// www.redalyc.org/pdf/422/42254536005.pdf.

23. Tabares JC, López YL. Salud y riesgos ocupacionales por el manejo de plaguicidas en campesinos agricultores, municipio de Marinilla, Antioquia, 2009. Rev. Fac. Nal. Salud Pública [internet]; 2011 [citado 2018 dic. 14]; 29(4):432-44. Disponible en: https://aprendeenlinea. udea.edu.co/revistas/index.php/fnsp/article/view/9473.

24. Restrepo M, Muñoz N, Day N, et al. Prevalence of adverse reproductive outcomes in a population occupationally exposed to pesticides in Colombia. Scand. J. Work. Environ. Heal. 1990;16(4):232-8.

25. Restrepo M, Muñoz N, Day N, et al. Birth defects among children born to a population occupationally exposed to pesticides in Colombia. Scand. J. Work. Environ. Heal. 1990;16(4):239-46.

26. Alcaldía de Medellín, "Perfil demográfico 2016-2020 para el Corregimiento 60. San Cristóbal" [internet] 2015 [citado 2018 may. 11]. Disponible en: https://www.medellin.gov. co/irj/go/km/docs/pccdesign/SubportaldelCiudadano_2/ PlandeDesarrollo_0_17/IndicadoresyEstadsticas/Shared\%20 Content/Documentos/ProyeccionPoblacion2016-2020/ Perfil\%20Demogr\%C3\%A1 fico\%202016\%20-\%202020\%20 Corregimiento\%2060_San\%20Cristobal.pdf.

27. Yadav IC, Devi NL, Syed JH, et al. Current status of persistent organic pesticides residues in air, water, and soil, and their possible effect on neighboring countries: A comprehensive review of India. Sci. Total. Environ. [internet]. 2015 [citado 2019 feb. 12]; 511:123-37. Disponible en: http://dx.doi.org/10.1016/j. scitotenv.2014.12.041.

28. Roberts JR, Routt Reigart J. Organophosphate insecticides. En: Recognition and Management of Pesticide Poisonings
Washington: Environmental Protection Agency [internet]; 2013 [citado 2019 jul. 2]. pp. 43-52. Disponible en: https://www.epa. gov/sites/production/files/2015-01/documents/rmpp_6thed_ final_lowresopt.pdf.

29. SajoR. Corregimientos de Medellín [internet] 2007 [citado 2018 nov. 3]. Disponible en https://commons.wikimedia.org/wiki/ File:Corregimientos_de_Medellin.png.

30. Colombia, Ministerio de Salud. Decreto 1843, por el cual se reglamentan parcialmente los Títulos III, V, VI, VII y XI de la Ley 09 de 1979, sobre uso y manejo de plaguicidas (1991 jul. 22).

31. Altieri MA, Nicholls CI. Scaling up agroecological approaches for food sovereignty in Latin America. [internet]. Developmen. 2008 [citado 2018 nov. 8]; 51(4):472-80. DOI: https://doi:10.1057/ dev.2008.68. Disponible en: https://www.researchgate. net/publication/23779494_Scaling_up_Agroecological_ Approaches_for_Food_Sovereignty_in_Latin_America.

32. Damalas C, Koutroubas S. Farmers' training on pesticide use is associated with elevated safety behavior. toxics [internet]. 2017 [citado 2019 ene. 10]; 5(3):19. DOI: https://doi.org/10.3390/toxics5030019. Disponible en: http://www.mdpi.com/2305-6304/5/3/19.

33. Colombia, Congreso de la República, Ley 101, Ley General de Desarrollo Agropecuario y Pesquero (1993 dic. 23).

34. Varona ME, Idrovo AJ. Determinantes sociales de la intoxicación por plaguicidas entre cultivadores de arroz en Colombia. Rev. Salud Pública [internet]. 2016 [citado 2018 dic. 4]; 18(4):61729. DOI: https://doi.org/10.15446/rsap.v18n4.52617. Disponible en: http://www.revistas.unal.edu.co/index.php/revsaludpublica/ article/view/52617.

35. Harrison J. Pesticide Drift and the Pursue of Environmental Justice. London: Massachusetts Institute of Technology Press; 2011.

36. Provost C, Gerber BJ. Political control and policy-making uncertainty in executive orders: The implementation of environmental justice policy. J Public Policy. [internet] 2019 [citado 2019 jun. 28]; 39(2):329-58. DOI: https://doi.org/10.1017/ S0143814X18000077. Disponible en: https://www.cambridge.org/ core/services/aop-cambridge-core/content/view/01F8E1BB65AC 836C6579062083DFBA7F/S0143814X18000077a.pdf/political_ control_and_policymaking_uncertainty_in_executive_orders_the implementation_of_environmental_justice_policy.pdf.

37. Pfeifer G. Pesticides, migrant farm workers, and corporate agriculture: How social work can promote environmental justice. J. Progress. Hum. Serv. 2016;27(3):175-90. DOI: https://doi.org/1 $0.1080 / 10428232.2016 .1196428$.

38. Arcury TA, Quandt SA, Dearry A. Farmworker pesticide exposure and community-based participatory research: Rationale and practical applications. Environ. Health Perspect. 2001;109(Supl. 3):429-34. DOI: https://doi.org/10.1289/ehp.01109s3429.

39. Kainga PE, Miller TA, Epidi TT. Assessment of awareness of benefits and hazards posed by agricultural pesticides to farmers in selected communities of Bayelsa State, Nigeria. Int J Res Agric For. 2016;3(2):32-40.

40. Richardson MJ, Madrigal DS, Wilkie A, Wong M, Roberts E. Environmental health tracking improves pesticide use data to enable research and inform public health actions in California. J. Public. Heal. Manag. Pract. 2017;23(5):S97-104. DOI: https://doi. org/10.1097/PHH.0000000000000595.

41. Gonzalez CG. Environmental justice, human rights, and the global south. Santa Clara J. INT'L L. [internet]. 2015 [citado 2018 oct. 22]; 13(151):151-195 Disponible en: https://digitalcommons.law. seattleu.edu/cgi/viewcontent.cgi?article $=1631 \&$ context=faculty. 